\title{
Factors influencing adherence to vacuum-formed retainer wear: A qualitative study
}

\begin{tabular}{|c|c|}
\hline Journal: & Journal of Orthodontics \\
\hline Manuscript ID & JOO-19-0022.R1 \\
\hline Manuscript Type: & Original Manuscript \\
\hline Keywords: & $\begin{array}{l}\text { Quality of life and orthodontics < Health services \& quality of life } \\
\text { aspects, Retention/retainers/stability, Health services \& quality of life } \\
\text { aspects }\end{array}$ \\
\hline Abstract: & $\begin{array}{l}\text { Objective: To explore factors influencing adherence to vacuum-formed } \\
\text { retainer wear over a minimum period of } 4 \text { years. } \\
\text { Design: A qualitative study based on a randomised controlled trial } \\
\text { assessing the effectiveness of orthodontic retainers. } \\
\text { Setting: Institute of Dentistry, Queen Mary University of London. } \\
\text { Participants: Fifteen participants wearing vacuum-formed retainers for at } \\
\text { least } 4 \text { years. } \\
\text { Methods: One-to-one semi-structured interviews were undertaken on a } \\
\text { criterion-based purposive sample of participants wearing vacuum-formed } \\
\text { retainers. The interviews were audio-recorded, transcribed verbatim and } \\
\text { analysed using Framework Methodology. } \\
\text { Results: High self-reported levels of adherence in the early stages of } \\
\text { retention were linked to a desire to maintain orthodontic outcomes and } \\
\text { the negative perception of potential post-treatment changes. However, } \\
\text { adherence typically reduced over time due to a combination of factors } \\
\text { including the negative impact of retainers on quality of life and pragmatic } \\
\text { issues related to retainer wear. Network support was found to be } \\
\text { important in the short- and long- term, with instances of self-directed } \\
\text { wear and negative beliefs concerning the importance of retainer wear } \\
\text { and predisposition to post-treatment changes. Lack of follow-up } \\
\text { appointments and immaturity of participants prompted independent } \\
\text { decisions to cease retainer wear. } \\
\text { Conclusions: Six key influencers of prolonged adherence with vacuum- } \\
\text { formed retainer wear were identified. Future strategies to improve } \\
\text { adherence should account for these while also being responsive to time } \\
\text { elapsed since debond and patient age. }\end{array}$ \\
\hline
\end{tabular}

\section{SCHOLARONE Manuscripts}




\section{Abstract}

Objective: To explore factors influencing adherence to vacuum-formed retainer wear over a minimum period of 4 years.

Design: A qualitative study based on a randomised controlled trial assessing the effectiveness of orthodontic retainers.

Setting: Institute of Dentistry, Queen Mary University of London.

Participants: Fifteen participants wearing vacuum-formed retainers for at least 4 years.

Methods: One-to-one semi-structured interviews were undertaken on a criterion-based purposive sample of participants wearing vacuum-formed retainers. The interviews were audio-recorded, transcribed verbatim and analysed using Framework Methodology.

Results: High self-reported levels of adherence in the early stages of retention were linked to a desire to maintain orthodontic outcomes and the negative perception of potential post-treatment changes. However, adherence typically reduced over time due to a combination of factors including the negative impact of retainers on quality of life and pragmatic issues related to retainer wear. Network support was found to be important in the short- and long- term, with instances of self-directed wear and negative beliefs concerning the importance of retainer wear and predisposition to post-treatment changes. Lack of follow-up appointments and immaturity of participants prompted independent decisions to cease retainer wear.

Conclusions: Six key influencers of prolonged adherence with vacuum-formed retainer wear were identified. Future strategies to improve adherence should account for these while also being responsive to time elapsed since debond and patient age.

\section{Keywords}

Keywords: Vacuum-formed retainers, patient-clinician relationship, qualitative methodology, patient perspectives, compliance 


\section{Introduction}

Post-treatment changes following orthodontic treatment may lead to reduced patient satisfaction, compromised aesthetic outcomes and the potential burden of re-treatment (Kearney et al., 2016). As such, effective retention strategies are a priority for both clinicians and patients. Notwithstanding this, post-treatment changes following orthodontics is commonplace and has variously been regarded as the most challenging aspect within orthodontics (Oppenheim, 1934). Removable retention places a significant onus on patient adherence both in the short- and long- term in order to mitigate against true relapse and physiological recovery, allied to characteristic, age-related changes (Horowitz and Hixon, 1969).

Adherence with orthodontic retainers, unlike other removable appliances, is characterised by the long-term requirement of wear. However, only one-third of participants reported satisfactory wear levels of vacuum-formed retainers after 2 years despite being uniformly $(100 \%)$ adherent at 6-month follow-up (Al-Moghrabi et al., 2018). Motives for wearing removable functional appliances include the ability to see positive dental changes, allied to parental involvement and professional encouragement at follow-up appointments (Čirgić et al., 2015; El-Huni et al., 2019). However, it would be intuitive to expect facilitators of retainer wear to be different as these perform a relatively passive function in maintaining treatment outcomes with participants also having less frequent follow-ups.

Several studies have included assessment of factors relating to suboptimal adherence with removable orthodontic retainers including age, gender, time elapsed since debond, type of retainer and wear regimen, place of treatment and type of health insurance (Hichens et al., 2007; Pratt et al., 2011; Schott et al., 2013; Tsomos et al., 2014). Patients' attitudes towards their retainers, in terms of whether they are considered an "investment" or a tedious commitment, seem to be an important determinant of adherence levels (Kearney et al., 2016). Discomfort, hassle, embarrassment and aesthetics concerns with orthodontic retainers are commonly reported (Bennet and Tulloch, 1999; Wong and Freer, 2005; Hichens et al., 2007; Pratt et al., 2011). Failure to wear retainers has also been attributed simply to forgetting to wear them in $50 \%$ of patients (Lin et al., 2015).

Studies focusing on experiences with removable orthodontic retention have been limited to questionnaire-based evaluation and content analyses of social media posts (Hichens et al., 2007; Pratt et al., 2011; Al-Moghrabi et al., 2017; Forde et al., 2017). Although questionnaires provide measurable effects, they do not provide sufficient depth and understanding of the implication of retainer-related experiences. In particular, questionnaires may be based on what clinicians think is important, which may result in pertinent issues being overlooked. Qualitative methods to gather detailed information about patients' experiences during the retention phase that cannot be satisfactorily answered otherwise are therefore required. The aim of this study was therefore to explore factors influencing removable retainer wear giving an in-depth understanding of patient perspectives. 
Ethical approval was obtained (XXXXXXXXXXXXXX). A criterion-based purposive sample of participants wearing vacuum-formed retainers was recruited from a previous randomised controlled trial (AlMoghrabi et al., 2018) including both males and females and reflecting a variety of adherence levels. Participants attending a 4-year follow-up research appointment were provided with a patient information sheet and invited to take part in the interviews. The interviews were undertaken in a nonclinical area in the XXXXXXXXX.

A topic guide was developed and piloted based on professional opinion ( $X X, X X X, X X X)$ (Appendix). The topic guide was adapted throughout the study based on participant responses. The interviews involved discussions about the different phases of treatment following a chronological order from appliance debond, retainer fit visit, and the different phases of retention including experiences of fullfollowed by night- time wear for long term. Questions pertaining to understanding of retainers, facilitators and barriers to retainer wear, the process of seeking information during retention phase, opinion about follow-up appointments and being discharged were also asked. Prompts were used to facilitate an in-depth understanding of responses (Ritchie et al., 2013). Relevant demographic and clinical data were obtained. Subjective data concerning duration of retainer wear was recorded during the interview.

The interviews were carried out by an orthodontic PhD student (XX), who undertook formal qualitative research training prior to commencing the study. The first 3 interviews were undertaken in the presence of a qualitative researcher (XX). The participants were informed about the interviewer's research and clinical roles prior to the interview. Interviews were audio-recorded and transcribed verbatim, and lasted between 45 and 101 minutes. Transcription commenced directly after the interview using a transcription service, and data were anonymised for analysis. The interviewer reviewed the accuracy of all transcripts prior to the analysis stage. Field notes were taken and were referred to during data analysis.

Data were analysed by two authors ( $X X$ and XXX) using NVivo ${ }^{\mathrm{TM}}$ qualitative data analysis software (QSR international Pty Ltd, Australia, Version 11) following the Framework Methodology involving: familiarisation, identification of thematic framework, indexing, charting, mapping and interpretation (Ritchie et al., 2013). Two researchers (XX and XXX) cross-checked the emergent themes in parallel with data interpretation to ensure validity of the findings. Any disagreements were resolved by joint discussion (XX, XXX, XXX). 


\section{Results \\ Participant characteristics}

A total of fifteen participants wearing vacuum-formed retainers $\left({ }^{\left({ }^{1} 1-\mathrm{p} 15\right.}\right)$ were interviewed including 10 females $(F)$ and 5 males $(M)$ ranging from 19 to 30 years (Table 1). Average years in retention was 4.8 (range: 4 to 5.6 years). Six refused to take part in the interviews due to time commitment. Selfreported levels of adherence ranged from few weeks (W), few months (M) to adherent throughout.

\section{Effect of time and age}

Six main themes influencing retainer wear were modified mainly by time elapsed since debond and participant age (Figure 1). Generally, retainer wear fluctuates and typically wanes over time, in some cases ceasing altogether. Initially, participants were motivated as a result of excitement about orthodontic treatment outcomes as they were eager to maintain the results "short term satisfaction" being linked to a novelty effect. However, motivation appears to diminish over time as inconveniences and pragmatic issues related to orthodontic retainers take hold. The follow-up appointments typically reduce over time and ultimately cease, partly explaining the associated reduction in adherence. After several months, most participants went through a phase of questioning the need to wear the retainers often making independent decisions to taper or cease wear. If post-treatment changes arose, selfmanagement and prescription were reported. Nevertheless, participants also referenced occasions in which they improved relaying the factors that prompted this. When reflecting on their experiences, participants repeatedly referred to their immaturity at the start of retention as a barrier to retainer wear.

\section{Theme 1. Beliefs concerning retention}

Retainers were considered important in maintaining post-treatment outcomes being synonymous with feeling "secured" and likened to a "safety net". Retainer wear was seen as a manifestation of a "perfectionist" and a reflection of taking care of oneself. However, some confusion arose when poor adherence did not necessarily result in noticeable post-treatment dental changes leading participants to question the importance of retainer wear.

"[A friend[has stopped wearing the retainers. And their teeth hasn't really changed as much. I started thinking that maybe it's not that big of a deal to stop wearing them." (M-Adherent to $\left.1 Y^{p 1}\right)$

Questioning the need for the retainers stemmed from their passivity in maintenance of posttreatment outcomes. However, their importance became appreciated once dental changes were noticed stimulating attempts to correct post-treatment changes. Furthermore, retainers were not perceived as a continuation of treatment in all cases, with debond symbolising the end of treatment.

"You don't really see what it's doing because your teeth are straight and the retainers are just a cover. It's only once the damage has been done." (M- Adherent to 6Mon ${ }^{p 4}$ )

"I was strict with myself, but then later on I was just a bit like 'The treatment's already done. I don't need to continue." (F- Adherent to 2Mon ${ }^{\text {P15) }}$

Patients' beliefs about their predisposition to post-treatment changes dictated adherence levels. For some, feeling at lesser risk created a barrier to retainer wear. For example, having particularly (1) long duration of orthodontic treatment resulted in questioning the need to wear the retainers. The (2) mild nature of pre-treatment malocclusion made some participants feel less susceptible to posttreatment dental changes. Participants justified the reduction in adherence over time as the (3) time 
elapsed since debond increased. Following debond, participants felt greater susceptibility to posttreatment dental changes, resulting in high adherence levels to allow teeth "to settle". However, after a period of time, the perceived need for the retainers diminished resulting in poorer adherence.

"After one year, your teeth have become more fixed in that position, so they are less likely to move." (F-Adherent to 2YP2)

The long-term requirement for retainers was viewed as an unrealistic "commitment" by participants. The length of retainer wear was linked to a certain age in which dental aesthetics may be a lesser priority. Furthermore, the inability to remember the details of the recommendations made in terms of duration and length of retainer wear was evident.

"Once you've reached that stage where you don't really care how people see you anymore, then why are you going to carry on wearing your retainers?" (M- Adherent to 6Mon ${ }^{p 4}$ )

\section{Theme 2. Perceived negative impact of post-treatment changes}

Post-treatment dental changes were associated with a number of negative connotations. Reluctance to undergo orthodontic retreatment due to pain and impaired smile aesthetics was a motive for wearing retainers. Financial burden associated with fixed orthodontic retreatment was also a stimulus for retainer wear.

Participants were eager to prevent post-treatment dental changes when reflecting on their previous experiences with fixed appliances. Jeopardising orthodontic treatment outcomes was regarded as a devaluation of time and commitment previously invested in prolonged orthodontic treatment. The adherence early in the retention phase appears to reflect the investment involved.

"Four years-worth of braces treatment that I cannot let go down the drain and one surgery... I didn't wear braces to my prom for no reason." (F-Adherent throughout ${ }^{P 5}$ )

\section{Theme 3. Effect on quality of life}

The negative effects of retainers on quality of life seem to inhibit retainer wear despite participants expressing the desire to be adherent. Negative experiences were related to physical and social impacts with perceived burden on the participants' lives. In relation to physical impact, pain markedly constrained retainer wear, particularly after a period of non-adherence due to hassle or forgetfulness, where participants reported experiencing numbness and discomfort. However, for some participants, retainer tightness prompted increase or resumption of retainer wear.

"When I didn't wear it one time, my teeth were tight. So I was like, 'I need to make sure that I wear it afterwards.' That just gave me the push that I need to wear it." (M-Adherent throughout ${ }^{\text {p11) }}$

Participants' response to pain varied with some open to tolerating pain to allow dental settling. However, in extreme cases, increased burden due to pain led to a choice being made to completely stop retainer wear.

"It hurts but I don't really mind because I know it's going to keep my teeth straight." (FAdherent to $2 Y^{P 2}$ )

"I gradually stopped wearing them, so when I put them back on, they really hurt. And then, I'd be less likely to wear it. Until I just stopped wearing it." (F-Adherent to 2Mon ${ }^{\text {P15) }}$ 
During retainer wear, participants felt conscious of having "something foreign", an "extra layer" and "extra heaviness" on their teeth. Others expressed the feeling of "lack of freedom" during retainer wear. In terms of appearance, although retainers are subtle, concerns were raised in this respect. Participants referenced occasions where retainer wear during the day caused embarrassment, impaired speech and had negative effects on social activities.

"When I took it out, I've heard 'That's nasty' because they've seen like a whole string of [saliva]. No matter how much you try to hide it, you can't." (F-Adherent throughout ${ }^{p 5}$ )

"Sometimes you'll miss a snack' because you don't want to take it out in front of people. You'd just rather eat at home." (F-Adherent throughout ${ }^{P 5}$ )

\section{Theme 4. Self-directed wear}

At the start of the retention phase, participants expressed intentions to wear retainers due to high motivation and excitement in relation to recent orthodontic treatment outcomes. However, participants perceived maintaining a habit of retainer wear on regular basis as a "chore", "time consuming" and a "constant effort". Maintaining the habit of retainer wear was challenging due to forgetfulness or laziness in some instances. A strategy of linking retainer wear to tooth-brushing time to overcome forgetfulness was described. The response of participants after missing a few days of retainer wear varied; in some, it culminated in longer period of non-adherence but others compensated with improved retainer wear thereafter.

"Just thinking, 'What's one day?' But then when you keep doing it, it's not just one day anymore. It's a week that's gone by that you haven't worn it a single day." (F- Adherent to $\left.6 W^{P 13}\right)$

"If I forgot to wear it for four hours, I'll keep it in for four hours extra because I knew that I didn't wear it the day before." (F-Adherent to $7 \mathrm{Mon}^{\mathrm{PG}}$ )

As participants were in long-term retention, those who were non-adherent felt that it would be challenging to set a habit to wear the retainers at this stage, with the importance of setting habits early in the retention phase expressed. Furthermore, participants alluded to the required hours and duration of wear being an additional challenge in the long-term.

"When I can't meet that unrealistic standard, I just give up." (F-Adherent to 2MonP15)

Taking an active role in the retention phase was evident in terms of self-diagnosis, self-prescription, and making uninformed choices without seeking professional advice. Participants attributed this to lack of follow-up appointments and supervision, as well as immaturity. Independent decisions in the form of self-prescription, and self-management of post-treatment dental changes were reported:

"I didn't think that I really needed to wear it [retainer] as often as they said, so I just went my own way... That was my own personal decision. I didn't need to consult them because it was my retainers, my teeth... I don't think it's normal for you to contact your orthodontist to tell them how you are using the retainers." ( $F$ - Adherent to $2 Y^{P 2}$ )

Although cessation of retainer wear was an independent decision in many instances, some participants reported not being conscious of the implications of this due to their age and immaturity:

"I was young, If I was ten years older then I would be a lot more aware of it.." (F- Adherent to $2 Y^{P 3}$ ) 
"I was a kid. I was lazy, I just took it with the flow. But now, I'm much more a planner." (MAdherent to $1 Y^{\mathrm{P} 10}$ )

\section{Theme 5. Network support}

The importance of parental and peer support was widely discussed by participants with regular referencing of parental advice about retainer wear; however, this did not necessarily translate into optimal wear.

"My Mum pushed me to wear the retainers. She said that I would have to listen to the doctor and I'm like 'Okay, I'll wear them'. But, I just couldn't.. I felt like it wasn't helping me in any way." (F- Adherent to 2Mon ${ }^{\text {P12) }}$

Peers and past patient experiences were quite powerful in raising awareness about the importance of retainers. For example, knowing a friend who experienced post-treatment dental changes because of poor adherence was a motivator to retainer wear. Seeking non-expert advice was reported from

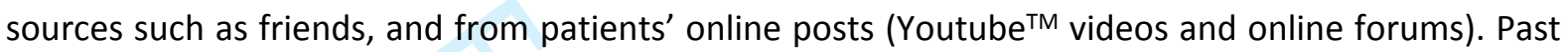
patient experiences seemed to resonate more than professional advice:

"I would ask my friends about how often they wore their retainers to see if maybe I should be wearing them more." (F-Adherent to $2 Y^{P 2}$ )

"It was people my age showing you how to clean them and that's what really attracted me, rather than seeing a professional video with a dentist." (F-Adherent to 7Mon ${ }^{p 6}$ )

Having friends who complied with wearing retainers made it socially more acceptable to participants. However, in some instances, unfavourable comparisons were made with peers, which served as a barrier to retainer wear. Furthermore, being the only one wearing retainers was perceived as problematic.

"Many of them [friends] came off their retainers a lot quicker than I did. And they said 'oh, nothing would happen, It doesn't matter'.. And their teeth hasn't moved as much. So I started thinking maybe it's not that big of a deal to stop wearing them." (M-Adherent to $1 Y^{P 1}$ )

"I was the first one to wear them in school so it was really unusual. If I saw someone wearing them, I would've been okay." (F- Adherent to 2Mon ${ }^{\text {P12) }}$

In terms of patient-clinician relationship, participants expressed trust in the advice provided and adhering to professional recommendation was given as a reason to wear retainers. Furthermore, participants saw dentists as an authoritative figure reporting a sense of obligation or duty towards the treating clinician:

"It would have a bit more of a personal connection, because he's the one that put your braces on, you'd feel more likely to put the retainers on... You don't want to disappoint them." (MAdherent to $1 \mathrm{Y}^{\mathrm{P} 10}$ )

In terms of communication with the treating clinician, some participants felt unable to articulate their concerns, needs and preferences to their dentist in view of their age, change of clinician, and infrequent follow-up appointments.

"I was a child... I wasn't as vocal to speaking out about what the problems are. If I had retainers now, I would be more opened to discussing it." (F-Adherent throughout ${ }^{p 5}$ ) 
"The appointments were so far and few between... I don't feel like I ever had a relationship with the dentist." (F-Adherent to 2Mon ${ }^{\text {P15) }}$

Participants valued the importance of follow-up appointments and linked this to the need to persevere with retainer wear. The lack of follow-up appointments was cited as a reason for cessation of wear in some. Furthermore, participants highlighted the importance of follow-up appointments in sharing concerns with the clinician, consolidating their knowledge of retainer wear and asserting the importance of retainers. At follow-up appointments, participants described wanting their clinician to show empathy, be friendly but also professional. Being discharged was viewed negatively, with participants described themselves as "on their own" without professional input.

"I didn't have any more appointments. So, I just wore it for one year and then stopped completely." (M-Adherent to $\left.1 \mathrm{Y}^{\mathrm{p} 10}\right)$

"It [a concern about retainers] wasn't something that I could speak to my dentist. Because I had no appointment, I had to Google it." (F-Adherent throughout ${ }^{14}$ )

"If I was to have my old dentist, I would have taken it [retainer wear] on board... He was so serious about it and had so much authority in my eyes. I took his words literally. The way he said it, it was words of encouragement but words of firmness." (F- Adherent to 7Mon ${ }^{p 6}$ )

Participants emphasised the importance of being informed about retainers from the start of the treatment in order to be prepared for the retention phase. One participant described being anxious prior to retainer fit expecting it to be a "big head brace". Participants felt that verbal communication was inadequate and must be supplemented with illustration of consequences of poor retention. Seeing photos of real patients was perceived as "evidence", "confirmation" and would "strike home".

"Even when my doctor gave me a booklet about retainers, I just read the first page... No one's bothered to read long booklets. You want video clips." (F-Adherent to 2Mon ${ }^{p 12}$ )

\section{Theme 6. Pragmatic issues}

Maintenance requirements including the limited durability and ease of loss due to size and transparency of the vacuum-formed retainers were both considered barriers to retainer wear especially in the long term. Incidents of retainer loss or breakage after a period of wear marked the end-point of the retention phase in many instances. Furthermore, participants reported challenges in terms of cleaning the retainers especially in accessing the fitting surface of teeth.

"Given one pair and being told that it is supposed to last for life is very impractical. Perhaps two pairs, like 1 pair and then a spare one. If anything happens they have a back-up." (FAdherent to $6 \mathrm{Mon}^{\mathrm{P7}}$ )

Inaccessibility to retainers also appeared to influence retainer wear with participants wearing the retainers typically if they were at home. However, change of setting complicates access with holidays and outdoor activities being associated with suboptimal wear. Visually seeing the retainer acted as a direct aide memoir to remind patients to wear the retainers in many instances, although this did not necessarily result in a decision to wear the retainers.

"I had a school trip and I just left it [retainers] at home. I didn't want the extra responsibility if I lost them..." (M-Adherent to $\left.1 Y^{p 10}\right)$ 


\section{Discussion}

This is the first study to explore patients' perspectives on factors influencing removable retainer wear allowing in-depth understanding of their experiences. A number of previously underreported issues related to prolonged retention were identified, for example, self-directed wear, reticence to share concerns with treating clinicians, negative influence of peers, negative beliefs, and unmet needs in terms of follow-up appointments and information provision.

Orthodontic retainers are theoretically a lifelong experience and are prescribed routinely following orthodontic treatment; however, relatively few studies have considered patients-reported outcomes in long-term retention (Pratt et al., 2011). Furthermore, most studies measure outcomes relevant to patients' experiences using questionnaires, risking failure to capture an in-depth response. Patients' experiences assessed in previous prospective studies primarily involved aspects related to discomfort, embarrassment, speech impairment and aesthetics (Hichens et al., 2007; Forde et al., 2017).

The qualitative approach assisted in understanding patients' behaviours, motives and impact of experiences related to treatment. Although quantitative studies offer an objective measure of different outcomes, in some instances explanation of such outcomes are only possible using qualitative methods. Therefore, the current study was carried out on a subset of the sample included in a previous randomised controlled trial (Al-Moghrabi et al., 2018).

Findings from a previous qualitative study have helped to inform the nature of a novel intervention designed to optimise adherence to intraoral elastic wear (Veeroo et al., 2014). Similarly, findings from this study can be utilised when developing interventions aiming to enhance retainer wear, support patients and addressing their needs. Furthermore, themes identified from the current study could be incorporated into measures of retainer impact on quality of life and overall experience. Moreover, participants reported preference for visual aids in information provision to allow better understanding of retainers and their importance. This reinforces previous work whereby visual images were used to highlight consequences of poor adherence to Hawley retainer wear (Lin et al., 2015). The latter in combination with verbal instructions and parental involvement resulted in higher adherence levels in comparison to verbal instructions alone (Lin et al., 2015). Relatively few (8\%) of prospective patients reported searching for online information related to their orthodontic treatment in a previous U.K.based study. ${ }^{17}$ However, as patients in long-term retention invariably lack formal follow-ups, more frequent use of Internet-based resources to access relevant information from non-professional sources would be intuitive (Al-Moghrabi et al., 2017). This also corroborates recent reports of patients sharing experiences and concerns about orthodontic retainers on social media (Al-Moghrabi et al., 2017), and was reflected in the frequent referencing of use of YouTube ${ }^{\mathrm{TM}}$ videos and other social media sites in the present analysis.

Participants included in this study were in prolonged retention and treated in a hospital setting by postgraduates in which patients are followed-up for up to a year post-debond. As such, the present findings may not be applicable to other settings. The recommended duration of retainer wear in this study was full-time for the first 6 months, followed by part-time (nights only wear). This approach was chosen as the initial clinical trial (O’Rourke et al., 2017) was designed to evaluate the comparative effectiveness of this wear protocol versus fixed retention. This regime is in line with previous research (Atack et al., 2007); notwithstanding this, nights only wear from the outset may be equally effective (Littlewood et al., 2016) and might risk less degradation and breakage of vacuum-formed retainers. Furthermore, participants' views may be potentially altered by response bias, especially given that interviewees were aware of the focus of the research. Notwithstanding this, the interviewer used nonleading questions and was not the treating clinician, potentially allowing participants to express their 
experiences more candidly. However, as the interviewer was an orthodontist, potential biased responses may stem from their preconceptions regarding the research topic. This was mitigated by the involvement of a qualitative researcher and by group discussions between the research team members during data collection and analysis.

\section{Conclusions}

Six key influencers of adherence with removable orthodontic retainer wear were identified. These include; beliefs concerning retention, perceived negative impact of post-treatment dental changes, effect on quality of life, self-directed wear, network support and pragmatic issues. The findings of this study highlight the need to develop interventions to improve the patient journey in the retention phase. Approaches to improving adherence should account for these while also being responsive to time since debond and patient age.

\section{Conflict of interest statement}

The authors declare that they have no competing interests.

\section{Acknowledgements}

We thank all participants who took part in the interviews.

\section{Funding}

This work was supported by the European Orthodontic Society; and XX's PhD is funded by the Saudi Arabian Cultural Bureau. 


\section{References}

Al-Moghrabi D, Johal A and Fleming PS (2017) What are people tweeting about orthodontic retention? A cross-sectional content analysis. American Journal of Orthodontics and Dentofacial Orthopedics 152(4): 516-522.

Al-Moghrabi D, Johal A, O'Rourke N, Donos N, Pandis N, Gonzales-Marin C and Fleming PS (2018) Effects of fixed vs removable orthodontic retainers on stability and periodontal health: 4-year follow-up of a randomized controlled trial. American Journal of Orthodontics and Dentofacial Orthopedics 154(2): 167-174.

Atack N, Harradine N, Sandy JR, Ireland AJ (2007) Which way forward? Fixed or removable lower retainers. The Angle Orthodontist 77(6): 954-959.

Bennett ME and Tulloch JC (1999) Understanding orthodontic treatment satisfaction from the patients' perspective: a qualitative approach. Clinical Orthodontics and Research 2(2): 53-61.

Čirgić E, Kjellberg H, Hansen K and Lepp M (2015) Adolescents' experiences of using removable functional appliances. Orthodontics and Craniofacial Research 18(3): 165-174.

El-Huni A, Colonio Salazar FB, Sharma PK, Fleming PS (2019) Understanding factors influencing compliance with removable functional appliances: A qualitative study. American Journal of Orthodontics and Dentofacial Orthopedics 155(2): 173-181.

Forde K, Storey M, Littlewood SJ, Scott P, Luther F and Kang J (2017) Bonded versus vacuum-formed retainers: a randomized controlled trial. Part 1: stability, retainer survival, and patient satisfaction outcomes after 12 months. European Journal of Orthodontics 40(4): 387-398.

Hichens L, Rowland H, Williams A, Hollinghurst S, Ewings P, Clark S, Ireland A and Sandy J (2007) Cost-effectiveness and patient satisfaction: Hawley and vacuum-formed retainers. European Journal of Orthodontics 29(4): 372-378.

Horowitz SL and Hixon EH (1969) Physiologic recovery following orthodontic treatment. American Journal of Orthodontics 55 (1): 1-4.

Kearney MK, Pandis N and Fleming PS (2016) Mixed-methods assessment of perceptions of mandibular anterior malalignment and need for orthodontic retreatment. American Journal of Orthodontics and Dentofacial Orthopedics 150(4): 592-600.

Lin F, Sun H, Ni Z, Zheng M and Yao L (2015) A feasible method to improve adherence of Hawley retainer in adolescent orthodontic patients: a randomized controlled trial. Patient Preference and Adherence 9: 1525-1530.

Littlewood SJ, Millett DT, Doubleday B, Bearn DR, Worthington HV (2016) Retention procedures for stabilising tooth position after treatment with orthodontic braces. Cochrane Database of Systematic Reviews. 2016(1).

Oppenheim A (1934) Crisis in orthodontia. Part 1 Tissue changes during retention. International Journal of Orthodontia 20: 639-644.

O'Rourke N, Albeedh H, Sharma P, Johal A (2016) Effectiveness of bonded and vacuum-formed retainers: a prospective randomized controlled clinical trial. American Journal of Orthodontics and Dentofacial Orthopedics 1:150(3): 406-415.

Pratt MC, Kluemper GT and Lindstrom AF (2011) Patient compliance with orthodontic retainers in the postretention phase. American Journal of Orthodontics and Dentofacial Orthopedics 140(2): 196-201.

Ritchie J, Lewis J, Nicholls CM and Ormston R. (2013) Qualitative research practice: A guide for social science students and researchers. Sage Publications; 2013.

Schott TC, Schlipf C, Glasl B, Schwarzer CL, Weber J and Ludwig B (2013) Quantification of patient compliance with Hawley retainers and removable functional appliances during the retention phase. American Journal of Orthodontics and Dentofacial Orthopedics 144(4): 533-540.

Stephens R, Ryan FS and Cunningham SJ (2013) Information-seeking behavior of adolescent orthodontic patients. American Journal of Orthodontics and Dentofacial Orthopedics 143(3): 303-309.

Tsomos G, Ludwig B, Grossen J, Pazera P and Gkantidis N (2014) Objective assessment of patient compliance with removable orthodontic appliances: a cross-sectional cohort study. The Angle Orthodontist 84(1): 56-61.

Veeroo HJ, Cunningham SJ, Newton JT and Travess HC (2014) Motivation and compliance with intraoral elastics. American Journal of Orthodontics and Dentofacial Orthopedics 146(1): 33-39. 
Wong P and Freer TJ (2005) Patients' attitudes towards compliance with retainer wear. Australian Orthodontic Journal 21(1): 45-53. 


\section{Figure caption:}

Figure 1. Factors influencing adherence to vacuum-formed retainer wear. 
Table 1. Participant characteristics $(n=15)$.

\begin{tabular}{|c|c|c|l|}
\hline $\begin{array}{c}\text { Participant } \\
\text { number }\end{array}$ & Gender & Age (years) & \multicolumn{1}{|c|}{$\begin{array}{c}\text { Self-reported adherence to VFR wear } \\
\text { throughout retention phase }\end{array}$} \\
\hline 1 & M & 22.5 & Adherent to $1 Y$ \\
\hline 2 & F & 19.6 & Adherent to $2 Y$ \\
\hline 3 & F & 21.7 & Adherent to $2 Y$ \\
\hline 4 & M & 20.5 & Adherent to 6Mon \\
\hline 5 & F & 21 & Adherent throughout \\
\hline 6 & F & 23.1 & Adherent up to 7Mon \\
\hline 7 & F & 21.7 & Adherent up to 6Mon \\
\hline 8 & F & 30.2 & Adherent up to 6Mon \\
\hline 9 & M & 20.5 & Adherent up to 2W \\
\hline 10 & M & 20.4 & Adherent up to $1 Y$ \\
\hline 11 & M & 20.2 & Adherent throughout \\
\hline 12 & F & 19.8 & Adherent up to 2Mon \\
\hline 13 & F & 19.6 & Adherent up to 6W \\
\hline 14 & F & 19.8 & Adherent throughout \\
\hline 15 & F & 24.7 & Adherent up to 2Mon \\
\hline
\end{tabular}

$\mathrm{M}=$ male; $\mathrm{F}=$ female; $\mathrm{VFR}=$ vacuum-formed retainer; $\mathrm{Y}=$ year; Mon: month; $\mathrm{W}=$ week 


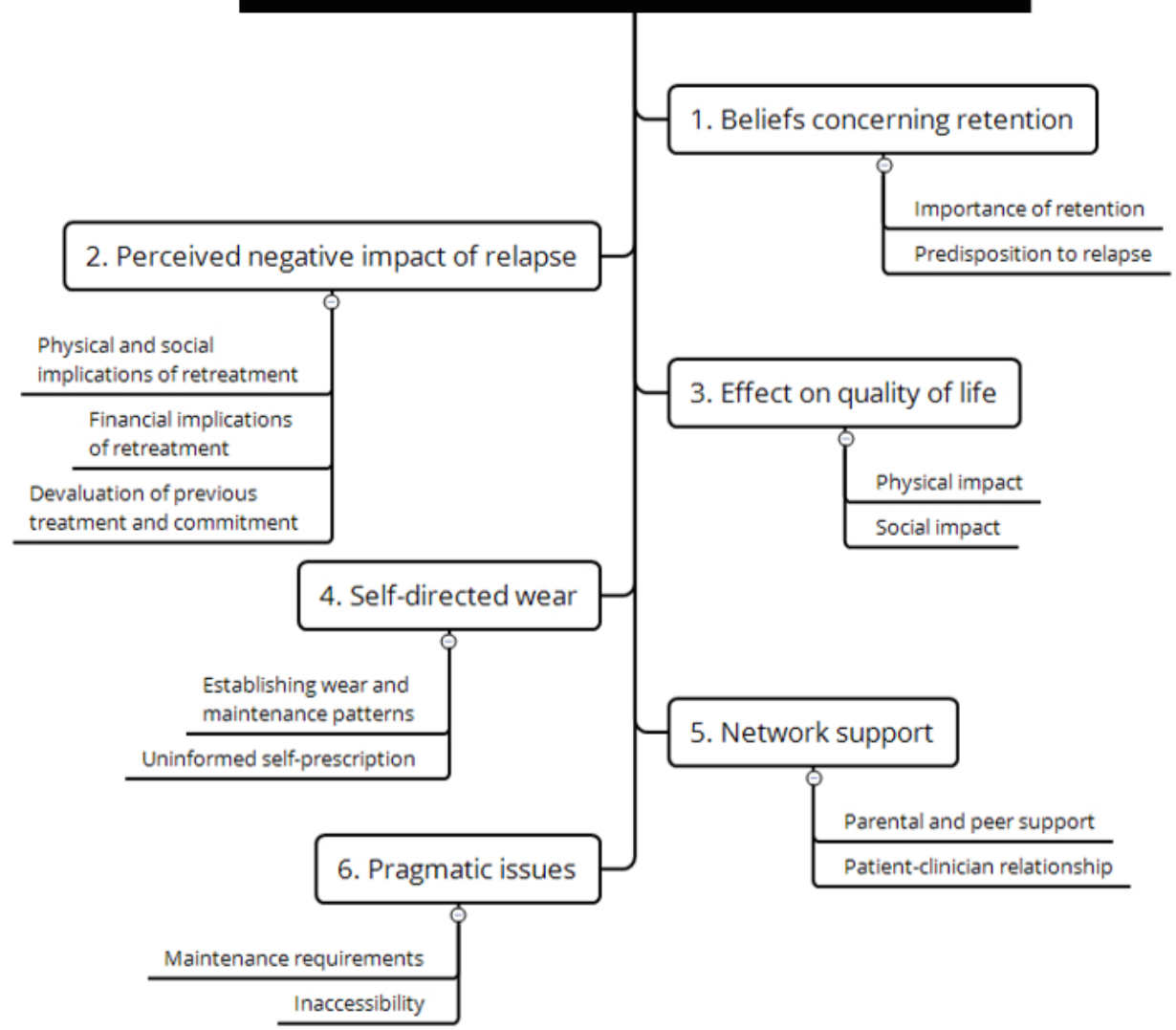

Figure 1. Factors influencing adherence to vacuum-formed retainer wear.

$$
123 \times 122 \mathrm{~mm}(150 \times 150 \mathrm{DPI})
$$




\title{
Topic guide
}

\section{Introduction}

- Presenting myself and my role in the study.

- We are carrying out a study to explore the factors that make people wear their retainers more or less than others. We would like you to share your thoughts about your experience with the retainers you were asked to wear after your braces were removed.

- Please make sure you have read the information sheet and consent form and have signed it.

- The conversation we are about to start will be recorded using a digital recorder and you will be notified about the start and finish of the recording. However, your name, address or any identifiable information will be kept confidential and will not be published. Your participation is voluntary and you are free to stop me at any time during the conversation.

- It is important to note that there are no right or wrong answers.

- Do you have any questions before we start?

\section{[Turn on the recorder]}

\author{
Warm-up questions \\ - How old are you? \\ - What year are you in at school?
}

\section{Background information about getting braces}

- What was the problem with your teeth that made you see the orthodontist? Why did you get braces?

- What was it like having braces? 


\section{Factors influencing adherence to vacuum-formed retainer wear: A qualitative study}

\section{Day of debond}

- Tell me about the day when you the braces off? How did it feel?

- What did you think of your teeth at the end?

- What did your family and friends think of the results? How did it make you feel?

- What advice was given to you at that day? Any examples?

- Did you look for any advice from other sources? If yes, why, and what were these sources.

\section{$\underline{\text { Retention phase }}$}

- What do you think of your retainers?

- How did it feel when you had your retainers at the start?

- Tell me more about your experience with the retainers when you had to wear them for fulltime/part-time.

- [Prompt] If the participant reported wearing their retainers: What makes you wear them? Can you give me some examples?

- [Prompt] If the participant reported not wearing the retainers: What makes you not wear them?

- [Prompt] If the participant mentions forgetting to wear the retainers: When do you usually forget? Why do you think that is? Can you tell me some examples?

- What do you understand about having to wear the retainers "long-term"? What are your thoughts about it?

\section{Conclusion}

- Thank you for your time.

- As we mentioned before, this interview is confidential and will be anonymous.

- The information taken from this interview will help us understand facilitators and barriers to retainer wear.

- That was everything I wanted to ask you. Is there anything else you would like to add/ask me?

\section{[Turn off the recorder]}

